\title{
CÁLCULO DA DISPONIBILIDADE E POSIÇÃO NO CICLO DE VIDA DE TRÊS LINHAS DE PRODUÇÃO DE UMA EMPRESA DA INDÚSTRIA QUIIMICA
}

\author{
Olívio Silva Filho \\ Miguel Afonso Sellitto ${ }^{2}$
}

\begin{abstract}
Resumo : O objetivo deste artigo foi calcular a disponibilidade $A v(t)$ e identificar a posição no ciclo de vida de três linhas de produção de BOPP (Biaxially Oriented Polypropylene Polipropileno Biorientado). Por software, foram modelados os tempos até a falha e os tempos até o reparo de vinte eventos de falha para cada linha, ocorridos em abril de 2012. As distribuições exponencial e de Weibull modelaram os $T B F$ e as distribuições normal e lognormal modelaram os TTR. Pelos valores esperados das distribuições, calcularam-se as disponibilidades $A v(t)$, que ficaram entre $92 \%$ e $95 \%$. Pelos fatores de forma das distribuições dos TBF concluiu-se que as linhas 1 e 3 encontram-se no período de desgaste e a linha 2 no período de vida útil.
\end{abstract}

Palavras-chave: Confiabilidade, Manutenibilidade, Disponibilidade, RCM.

Abstract : The aim of this article was to calculate availability $A v(t)$ and to identify the position in the life cycle of three lines of production of BOPP (Biaxially Oriented Polypropylene Polypropylene). Supported by software, the time to failure and time to repair of twenty failure events were modeled for each line, occurred in April 2012. The exponential and Weibull distributions have modeled the TBF and normal and lognormal distributions modelled the TTR. By the expected values of the distributions, the calculated availability Av(t) ranged from 92 to $95 \%$. By distributions form factors of TBF concluded that lines 1 and 3 are in wear-out period and line 2 at useful life period.

Keywords: Reliability, Maintainability, Availability, RCM.

\footnotetext{
${ }^{1}$ Engenheiro Eletricista e Especialista em Gestão da Manutenção Industrial pela UNISINOS. E-mail: olivio.silva@comexigroup.com

${ }^{2}$ Professor e pesquisador do PPGEPS - UNISINOS, bolsista de produtividade do CNPq. E-mail: sellitto@unisinos.br
} 


\section{INTRODUÇÃO}

A manutenção, segundo a ABNT (1994), é a combinação de todas as ações técnicas e administrativas, incluindo supervisão, destinadas a manter ou recolocar um item em um estado no qual possa desempenhar uma função requerida. Esta capacidade do item desempenhar sua função alcança grande relevância quando o mesmo está inserido em um sistema complexo de produção, formado por várias etapas e com muitos mecanismos de transformação. Para garantir a qualidade e produtividade nas indústrias, a atuação de uma manutenção industrial é fundamental, onde a função não deve ficar resumida ao consertar ou reparar equipamentos no menor tempo possível, e sim manter o equipamento disponível para produção, reduzindo a possibilidade de paradas não planejadas (Pinto e Xavier, 1999).

Estratégias de manutenção podem ser importantes na gestão de atividades produtivas. Com ela, pode-se estender a vida útil dos equipamentos, aumentar sua disponibilidade e mantê-los em condições para a produção. A não realização desta manutenção, ou a falta de uma estratégia eficiente, pode ser responsável por falhas frequentes dos equipamentos, pelos atrasos na entrega da produção e pela diminuição da qualidade dos produtos, levando a empresa à diminuição de sua produtividade (Swanson, 2001).

Diante de um cenário onde as metas de produção são cada vez mais arrojadas, é imprescindível que os recursos e meios de produção estejam disponíveis com alta confiabilidade, permitindo que as máquinas realmente sigam produzindo e não parem por problemas de manutenção. Para que isto ocorra é necessário gerenciamento por meio de planejamento, escolha correta de estratégia e capacitação dos colaboradores. A função da manutenção industrial ganha maior destaque em processos contínuos e máquinas de grande porte, onde cada etapa do processo deve ser mantida para que a produção possa seguir acontecendo (Wuttke e Sellitto, 2008).

A gestão estratégica da manutenção permite focalizar apenas as ações que têm mais chances de trazerem bom resultado com baixo esforço. Ações que exigem alto esforço, tais como fazer corretivas em toda quebra; ações que oferecem pouco resultado, tais como fazer preditiva em toda máquina; ou ações contraproducentes, tais como fazer preventiva antes do desgaste, devem ser identificadas e abandonadas em uma gestão estratégica da manutenção (Sellitto, 2005).

O objetivo deste artigo é calcular a disponibilidade de três linhas de produção de BOPP (Película de Polipropileno Biorientada) em uma empresa da indústria química. O BOPP é um filme plástico usado em embalagens flexíveis, fitas adesivas, etiquetas e rótulos. Os objetivos secundários são modelar as funções confiabilidade e manutenibilidade, posicionar as linhas no ciclo de vida, e calcular as disponibilidades das três linhas estudadas. As principais técnicas de pesquisa foram a modelagem dos tempos entre falhas ( $T B F$ - time between failures) e tempos até o reparo (TTR - time to repair). No ano de 2012, estas linhas de produção pararam por emergência por mais de 2.500 horas. Em indústrias de processamento contínuo, a parada de equipamentos críticos representa a parada total da respectiva linha de produção (Sellitto, 2002). Não é objetivo do artigo analisar qualitativamente as causas para os tipos de falhas modelados nem estratégias para a gestão dos resultados encontrados. Optou-se por estudar comparativamente as três linhas de produção da empresa, devido a que as três linhas constituem a totalidade da capacidade produtiva da empresa.

Para a definição da estratégia de manutenção das linhas, modelaram-se os tempos até a falha pela distribuição de Weibull. $O$ fator de forma da distribuição ajustada identifica a posição da linha no ciclo de vida do equipamento. Foram utilizadas técnicas quantitativas da confiabilidade. $\mathrm{O}$ método de pesquisa foi a modelagem.

$\mathrm{O}$ artigo está organizado em: (i) referencial teórico dos conceitos de confiabilidade, manutenibilidade e distribuições de probabilidade; (ii) identificação dos equipamentos analisados e atual situação da manutenção; (iii) 
obtenção dos TTF e TTR das três linhas estudadas; (iv) apresentação dos resultados obtidos pela modelagem dos tempos coletados para linhas de produção e (v) análise dos resultados obtidos com considerações comuns aos três casos. Parte desta pesquisa foi financiada pelo $\mathrm{CNPq}$.

\section{REVISÃO}

A manutenção centrada em confiabilidade (MCC ou RCM - Reliability Centered Maintenance) pode contribuir para manter a funcionalidade de processos produtivos e equipamentos. A MCC tem como meta a identificação dos principais modos de falha e suas consequências, a escolha de técnicas e estratégias de manutenção e o acompanhamento da aplicação a fim de minimizar o risco e a severidade dos vários modos de falha. Uma das decorrências da MCC é o aumento da disponibilidade dos equipamentos e sistemas de produção. Apesar de ter sido criada na indústria aeronáutica, as técnicas da MCC são aplicáveis a outras indústrias (Siqueira, 2005; Sellitto et al., 2002).

A implantação da MCC exige tanto técnicas qualitativas como quantitativas. As primeiras estão voltadas mais ao estudo dos vários modos de falha e suas consequiências. As segundas modelam grandezas para o cálculo de medidas de desempenho, tais como tempos médios entre falhas (MTBF) e tempos médios até o reparo (MTTR) de equipamentos e sistemas industriais (Mendes, 2011). Para este artigo, interessa mais a abordagem quantitativa da MCC, principalmente a modelagem das funções confiabilidade e manutenibilidade.

A NBR 5462 é baseada na norma internacional IEC 50 (191) e define confiabilidade e manutenibilidade. $\mathrm{Na}$ NBR 5462, o termo confiabilidade está relacionado à função de probabilidade que descreve o tempo até a falha, também chamada de taxa de êxito e sinônimo da fiabilidade de um equipamento ou sistema. A manutenibilidade está relacionada à função de probabilidade que descreve os tempos até o reparo. Ambos os tempos são tratados como variáveis aleatórias. Seguem as definições completas, segundo a ABNT (1994):

- confiabilidade $R(t)$ : probabilidade de um item desempenhar uma função requerida, sob dadas condições, durante o intervalo de tempo $t$;

- manutenibilidade $M(t)$ : probabilidade de uma dada ação de manutenção, para um item sob dadas condições de uso, ser efetuada até o tempo $t$;

- disponibilidade $A v(t)$ : probabilidade de um item estar em condições de executar uma certa função em um dado instante ou durante um intervalo de tempo determinado, levando-se em conta os aspectos combinados de sua confiabilidade, manutenibilidade e suporte de manutenção, supondo que os recursos externos estejam assegurados.

\subsection{FUNÇÕES $R(t), M(t), A v(t)$}

A função confiabilidade $R(t)$ é a probabilidade de não haver falha entre $0 \mathrm{e}$ $t$. A função confiabilidade $R(t)$ trabalha associada a outras três funções (FOGLIATTO e RIBEIRO, 2009):

- probabilidade acumulada de falha $F(t)$ : é a probabilidade de haver falha entre 0 e $t$, ela é o complemento para 1 de $R(t)$, dada pela equação (1):

$$
F(t)=1-R(t)
$$

- densidade de probabilidade de falhas $f(t)$ : é a probabilidade de haver falha entre $[t+\Delta t]$. Se o tempo até a falha de um equipamento é uma variável aleatória, sua função densidade de probabilidade de falhas é dada pela equação (2):

$$
R(t)=1-\int f(\mathrm{t}) \mathrm{d} t
$$

- função taxa de falha $h(t)$ : é a probabilidade condicional da ocorrência de falha no intervalo de $t$ a $t+\Delta t$, dado que não houve falha até o instante $t$. Este é o limite da probabilidade que um item falhe em 
um intervalo que tende a zero, dado que tenha sobrevivido até o início do intervalo. A função taxa de falha $h(t)$ é homóloga à função taxa de risco $\lambda(t)$.

As quatro funções se relacionam e são importantes para o estudo dos mecanismos de falhas encontrados nos sistemas produtivos (Siqueira, 2005). O estudo matemático dos mecanismos de falha inicia pela modelagem da variação temporal da probabilidade de falha funcional do item, por unidade de tempo, representada pela função de distribuição de probabilidade dada pela equação (3):

$$
f(t)=\frac{d F(t)}{d t}
$$

A densidade de probabilidade de falhas $f(t)$ e a probabilidade acumulada de falha $F(t)$ entre dois instantes de tempo $t_{l}$ e $t_{2}$, relacionam-se como nas equações (4) e (5).

$$
\begin{gathered}
F(t)=\int_{-\infty}^{t} f(t) \cdot d t \\
F\left(t_{2}\right)-F\left(t_{1}\right)=\int_{t_{1}}^{t_{2}} f(t) \cdot d t
\end{gathered}
$$

Nestas expressões, a variável tempo $t$ pode ser substituída por outra variável que traduza o ciclo operacional da função, tal como quilômetros rodados ou ciclos de máquinas e que esteja relacionada à probabilidade de ocorrência de falhas. À manutenção interessa a probabilidade de que o item sobreviva a um dado intervalo (de tempo, ciclo, distância, etc.). Esta probabilidade de sobrevivência é dada por:

$$
R(t)=\int_{t}^{\infty} f(t) \cdot d t=1-\int_{-\infty}^{t} f(t) \cdot d t=1-F(t)
$$

As funções $R(t), \quad f(t)$ e $F(t)$ relacionam-se com a função de taxa de falha do equipamento, dada pela equação (7):

$$
h(t)=\frac{f(t)}{R(t)}=\frac{f(t)}{1-F(t)}=\frac{d \log R(t)}{d t}
$$

O $M T B F$ é o valor esperado para o tempo até a (ou entre) falha(s) e é dado pela equação (8).

$$
M T B F=\int_{0}^{\infty} R(t) d t=\int_{0}^{\infty} t . f(t) d t
$$

A função manutenibilidade $M(t)$ informa a probabilidade que, dado que ocorreu uma quebra, o reparo seja concluído até o tempo $t$. O valor esperado para o tempo até o reparo é o MTTR e pode ser usado como indicador de qualidade do serviço de manutenção. Seja $T$ o tempo necessário para reparar um sistema a partir do instante da falha. A função de densidade de probabilidade do tempo de reparo $m(t)$ pode ser definida pela equação (9), na qual $m(t)$ é a probabilidade que o reparo exija um intervalo de tempo entre $t$ e $[t+\Delta t]$ (Lewis, 1996).

$$
m(t) . \Delta t=P[t \leq T \leq t+\Delta t]
$$

A função distribuição para reparo $M(t)$ é dada pela equação (10). O tempo médio para reparo (MTTR) é dado por (11).

$$
\begin{aligned}
M(t) & =\int_{0}^{t} m(t) \cdot d t \\
M T T R & =\int_{0}^{\infty} t \cdot m(t) \cdot d t
\end{aligned}
$$

A função disponibilidade $A v(t)$ combina confiabilidade e manutenibilidade. $A v(t)$ aumenta com o tempo entre falhas e diminui com o tempo de reparo, dados por MTBF e MTTR e segundo a equação (12) (Lafraia, 2001).

$$
A v(t)=\frac{M T B F}{M T B F+M T T R}
$$

A qualidade do projeto do equipamento e de sua manutenção pode ser medida por $A v(t)$. Para melhorar $A v(t)$, duas abordagens são possíveis e complementares (Sellitto, 2005): 
- Aceita-se que haverá falhas e reduzse o MTTR por meio de técnicas de projeto voltadas à manutenibilidade;

- Reduz-se o número de falhas, o que aumenta o $M T B F$, por meio de técnicas de projeto voltadas à confiabilidade.
Segundo Siqueira (2005), a evolução da taxa de falhas de equipamentos complexos como os utilizados em sistemas de produção industriais obedecem a um padrão como o da Figura 1, conhecido como curva da banheira (bath-tube curve).

\subsection{A CURVA DA BANHEIRA}

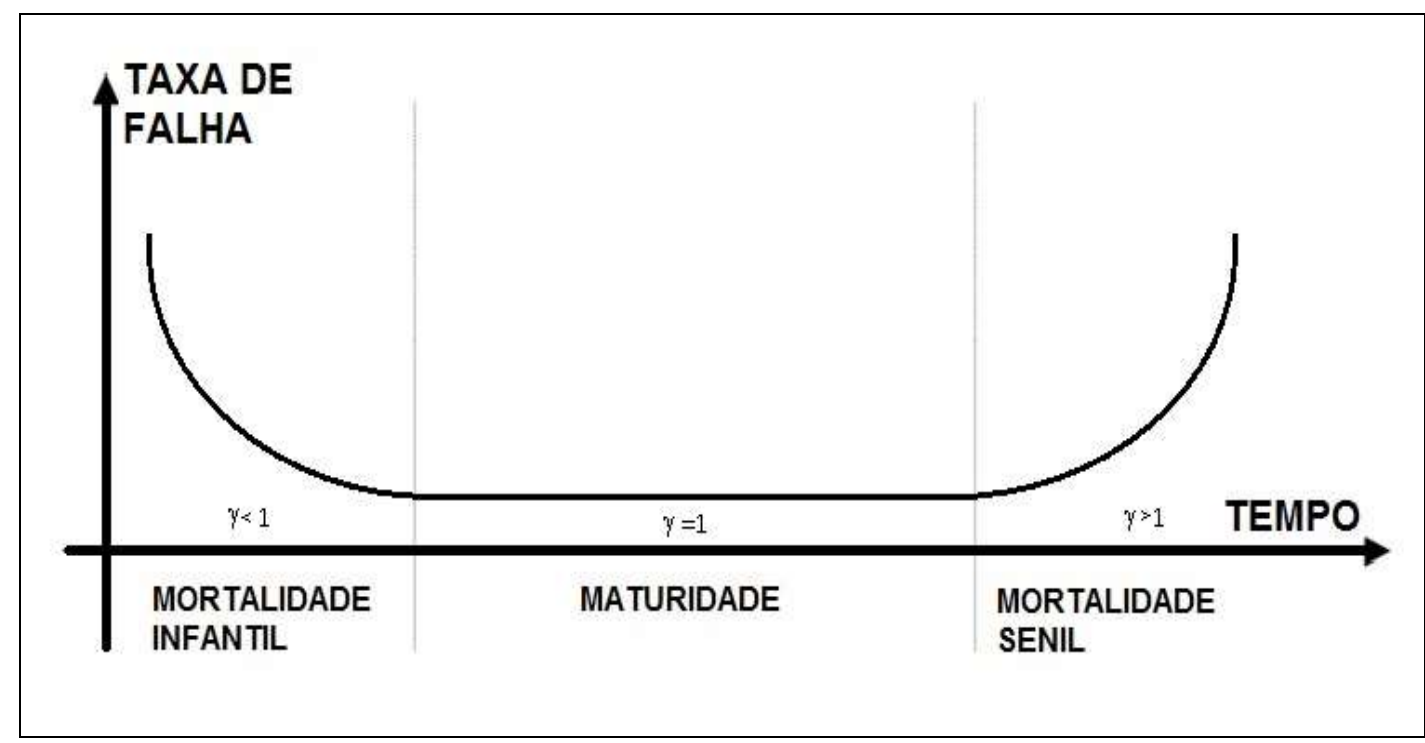

Fig. 1 - Curva da Banheira

Fonte: adaptado de Sellitto (2005) e Siqueira (2005)

A curva da banheira representa o comportamento da taxa de falha de um equipamento ou sistema produtivo ao longo do tempo. Na curva observam-se três fases características: mortalidade infantil, vida útil (ou fase de falhas aleatórias) e período de desgaste. Sellitto (2005) chama os dois últimos períodos de maturidade e mortalidade senil, termos que foram usados preferencialmente neste artigo. As fases estão associadas ao fator de forma $\gamma$ de uma eventual distribuição de Weibull que descreva a confiabilidade do equipamento.

No período de mortalidade infantil, a taxa de falhas é alta, porém decrescente. As falhas são preliminares e são causadas por defeitos ou fraquezas congênitas, erros de projeto, peças defeituosas, processos de fabricação inadequados, mão-de-obra destreinada, estocagem inadequada, instalação imprópria, partida deficiente, entre outras. A taxa de falhas diminui conforme são detectados e corrigidos erros de projeto ou de instalação, e conforme os reparos eliminam ou substituem itens frágeis ou com defeitos (Lafraia, 2001).

O período intermediário é a fase de maturidade. Nesta, o valor médio da taxa de falha é constante. As falhas ocorrem por causas aleatórias, geralmente externas ao equipamento. Falhas aleatórias podem ser de vários tipos: sobrecargas aleatórias, problemas de alimentação elétrica, vibração, impactos mecânicos, variações bruscas de temperatura, erros humanos, acidentes, impactos, mau uso do equipamento ou de matérias-primas, ou operação inadequada (Lafraia, 2001). Pela aleatoriedade envolvida, tais tipos de falha são de difícil controle.

$\mathrm{Na}$ última fase, há crescimento da taxa de falhas, o que representa o início do período final de vida do item. Esta fase é caracterizada pelo desgaste do componente, corrosão, fadiga, trincas, deterioração mecânica, elétrica ou química, manutenção insuficiente entre outros. Para 
produzir itens com vida útil mais longa, deve-se atentar para o projeto, utilizando materiais e componentes mais duráveis, um plano de inspeção e manutenção que detecte o início da mortalidade senil e a previna, por substituição preventiva de itens, reforma de sub-sistemas e eliminação de agentes nocivos presentes no meio produtivo (Lafraia, 2001).

O término da vida útil, sob o ponto de vista de confiabilidade, ocorre quando o item ingressa no período de mortalidade senil e não deve ser confundido com obsolescência mercadológica ou produtiva. $\mathrm{Na}$ obsolescência, o equipamento é substituído quando a função que desempenha perde o valor. No término da vida útil, o equipamento é substituído quando a sua confiabilidade em produzir o valor esperado cai abaixo do que se espera (Sellitto, 2005). Para Siqueira (2005), sistemas industriais evoluem na curva da banheira segundo várias dinâmicas, podendo ocorrer saltos, tais como a passagem direta da mortalidade infantil para a senil, ou a permanência por tempo indefinido em uma única fase, tal como ocorre com softwares.

Uma das principais funcionalidades associadas à curva da banheira está na escolha da estratégia de manutenção (Sellitto, 2005):

- Mortalidade infantil: a estratégia deve focar mais na correção ou ajustes do projeto e de instalação;

- Maturidade: a estratégia deve focar mais em ações preditivas ou de MPT, os programas de Manutenção Produtiva Total);

- Mortalidade senil: a estratégia deve ser preventiva, seguida de reforma, que recompõe o equipamento, eliminando as fragilidades devidas aos desgastes diversos.

\subsection{DISTRIBUIÇÕES PROBABILIDADE MANUTENÇÃO}

As distribuições de probabilidade de maior interesse em manutenção são: de Weibull, Exponencial, Normal, Lognormal e Gamma. A necessidade do uso de distribuições se explica pelo fato dos $T B F$ e TTR serem variáveis aleatórias. Mesmo que se mantenham as condições de trabalho, o tempo até a próxima falha ou o tempo até o reparo não são previsíveis. Suas probabilidades devem ser modeladas, se possível por distribuições conhecidas (Fogliatto e Ribeiro, 2009).

De modo geral, as distribuições explicam determinados tipos de estrutura de comportamento. No caso dos TBF, a distribuição de Weibull explica o comportamento dos tempos até e entre falhas para equipamentos em série, nos quais as causas competem entre si, ou seja, o primeiro item que falha é quem causa a falha geral do equipamento. A distribuição Gamma explica os TBF para equipamentos em paralelo: o último item que falha causa a falha geral. A distribuição exponencial é um caso particular da distribuição de Weibull quando o fator de forma é unitário, o que acarreta taxa de risco constante, ou seja, o equipamento não possui memória para o tempo até a falha. A distribuição lognormal explica os casos em que a falha se origina da multiplicação de um número de causas que tende a infinito. Por fim, a distribuição normal explica os casos em que a falha se origina da soma de um número de causas que tende a infinito. Para os TTR, se o reparo está relacionado a tarefas intelectivas ou estratégicas, tais como reparos complexos em sistemas integrados, é mais provável que a distribuição lognormal ajuste os tempos. Caso o reparo seja formado mais por atividades sequenciais, tais como a construção ou desmontagem de máquinas, é mais provável que a distribuição normal ajuste os tempos (Hahn e Shapiro, 1967; Lawless, 2003). Como premissa de pesquisa, Mengue e Sellitto (2013) adotaram a seguinte regra: (i) para os TBF, se Weibull ajustar os dados, as demais distribuições devem ser abandonadas; se a exponencial também ajustar os dados, a exponencial deve ser adotada; e (ii) para os TTR, se lognormal ou normal ajustarem os dados, as demais devem ser abandonadas.

A distribuição de Weibull é representada pela equação (13), na qual $\theta$ é o parâmetro de escala, $\gamma$ é o parâmetro de forma e $t$ é o tempo até a falha. 


$$
f(t)=\frac{\gamma}{\theta}\left(\frac{t}{\theta}\right)^{\gamma-1} \exp \left[-\left(\frac{t}{\theta}\right)^{\gamma}\right]
$$

O formato da função taxa de falha $h(t)$ é determinado pelo fator de forma $\gamma$, ao qual se dá a seguinte interpretação:

$-\gamma<1$ : taxa de falha decrescente, item em mortalidade infantil;

$-\gamma=1$ : taxa de falha constante, falhas aleatórias, zona de maturidade;

$-\gamma>1$ : taxa de falha crescente, falhas por fadiga ou mortalidade senil.

A distribuição Exponencial é um caso particular de Weibull quando $\gamma=1$. A distribuição ajusta falhas independentes que ocorrem por motivos aleatórios, os chamados processos de Poisson homogêneos. Ela descreve sistemas com taxa de falhas constante. É utilizada, preferencialmente, para tempo entre falhas aonde Weibull não consegue explicar os dados. A função distribuição de probabilidade é dada pela equação (14).

$$
f(t)=\lambda \cdot e^{-\lambda t}
$$

As distribuições normal, lognormal e gamma são dadas pelas equações de (15) a (17) (Lewis, 1996; Dodson e Nolan, 2002).

$$
\begin{aligned}
& f(t)=\frac{1}{\sigma \sqrt{2 \pi}} \exp \left[-\frac{1}{2}\left(\frac{t-\mu}{\sigma}\right)^{2}\right] \\
& f(t)=\frac{1}{\sigma t \sqrt{2} \pi} e^{\left[-\frac{1}{2} \ln \left(\frac{\mu-t}{\sigma}\right)^{2}\right]} \\
& f(t)=\frac{\alpha}{\Gamma(r)}(\alpha t)^{r-1} e^{-\alpha t}
\end{aligned}
$$

\section{A PESQUISA}

A disponibilidade $A v(t)$ foi calculada para três linhas de produção de $B O P P$ em uma empresa da indústria química. $\mathrm{O}$ processo produtivo é idêntico nas três linhas e os equipamentos foram fornecidos pelo mesmo fabricante. Entretanto existem características diferentes em cada uma das linhas, motivadas por opções do cliente e também pelo uso de tecnologia mais moderna na ocasião da montagem dos equipamentos mais recentes. A linha 1 iniciou atividades no ano 2000, a linha 2 em 2005 e a linha 3 em 2008. A Tabela 1 sintetiza as principais características dos equipamentos das linhas.

Tabela 1 - Características das linhas

\begin{tabular}{rccc}
\hline & LINHA 1 & LINHA 2 & LINHA 3 \\
\hline $\begin{array}{r}\text { Tempo de } \\
\text { Operação }\end{array}$ & 13 anos & 8 anos & 5 anos \\
Numero & 3 & 5 & 5 \\
$\begin{array}{r}\text { Camadas } \\
\text { Largura }\end{array}$ & $7,2 \mathrm{~m}$ & $8,2 \mathrm{~m}$ & $8,2 \mathrm{~m}$ \\
$\begin{array}{r}\text { Nominal } \\
\text { Capacidade }\end{array}$ & $2500 \mathrm{t} / \mathrm{h}$ & $4000 \mathrm{t} / \mathrm{h}$ & $4000 \mathrm{t} / \mathrm{h}$ \\
$\begin{array}{r}\text { Espessura } \\
\text { Mínima }\end{array}$ & $15 \mu \mathrm{m}$ & $12 \mu \mathrm{m}$ & $12 \mu \mathrm{m}$ \\
$\begin{array}{r}\text { Espessura } \\
\text { Máxima }\end{array}$ & $50 \mu \mathrm{m}$ & $50 \mu \mathrm{m}$ & $50 \mu \mathrm{m}$ \\
\hline
\end{tabular}

Fonte: banco de dados da empresa

As linhas 2 e 3 estão mais próximas entre si em características, equipamentos e arranjo de montagem. A linha 1 tem um arranjo menor que as outras duas linhas.

\subsection{O PROCESSO PRODUTIVO}

A Figura 2 apresenta um diagrama do processo produtivo que vale para as três linhas estudadas.

Os diferentes tipos de insumos que compõem o filme de $B O P P$ são dosados e pesados eletronicamente antes de serem fundidos e arrastados pelas Extrusora e Coextrusoras. Uma vez misturado e aquecido, o material passa por uma matriz plana que faz um filme grosso e estreito, chamado de cast. Logo que sai da matriz, o cast é rapidamente resfriado no rolo chamado Chill Roll. Uma vez solidificado, o cast passa pelo estiramento longitudinal no $M D O$, que é um caminho de rolos esticador do filme. Nesta etapa, o material passa por rolos aquecidos que proporcionam uma temperatura que possibilita que o cast seja esticado no sentido da máquina. Esta etapa do $M D O$ e a próxima do $T D O$ é que fazem o estiramento nos dois sentidos e caracterizam o nome do produto final: Polipropileno Biorientado (BOPP). 


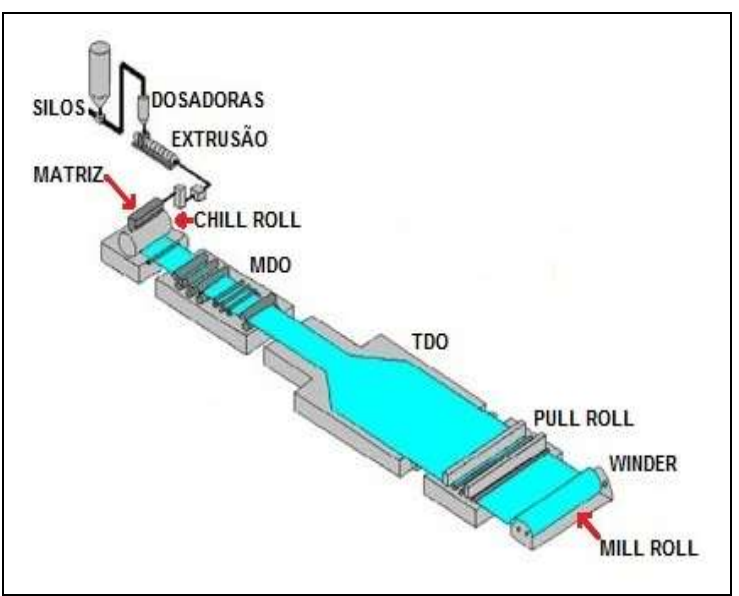

Figura 2: Diagrama do processo produtivo Fonte: adaptado de Bruckner (2013)

$\mathrm{Na}$ etapa do TDO, que é um alargador e definidor dimensional do filme,o cast é novamente aquecido, desta vez pela circulação de ar quente. O cast é preso nas duas pontas por pegadores transportados por corrente. À medida que o cast vai avançando para dentro do TDO, vai sendo esticado para os lados até atingir a largura desejada. Após um breve resfriamento o agora filme de BOPP passa pela etapa do Pull Roll que possibilita a retirada de aparas, medição da espessura e os tratamentos de superfície corona e chama. O filme é enrolado em um bobinador de fluxo constante, a Winder, que permite a troca automática dos eixos, sem interrupções na linha: enquanto um eixo é bobinado o outro pode ser retirado e preparado para assumir o bobinamento após o corte do filme. O produto final chama-se Mill Roll e pode ter largura máxima de $8,2 \mathrm{~m}$ e diâmetro de até 1.300 $\mathrm{mm}$.

Por esta descrição, pode-se perceber a quantidade de equipamentos que devem estar trabalhando sem apresentar falha para que o processo de fabricação do filme aconteça de maneira contínua. Desde o momento que se tem o material derretido na saída da Matriz plana, não podem ocorrer falhas em equipamentos. Neste percurso, até a Winder, não existem áreas para estocagem temporária do material produzido e as partes que chegarem ao fim do processo devem ser descartadas.

Trata-se de um processo em que as interrupções causam expressivo prejuízo. Uma vez que a passagem de material teve início, gasta-se tempo até ter-se a especificação adequada. Interrupções, portanto, implicam expressivo desperdício de matéria prima. O principal parâmetro ajustado é a espessura do filme, medida em micra. Por características do processo, o ajuste deve ser feito com o filme passando em velocidade nominal. Como o custo da matéria prima é alto, a parada da linha tem custo elevado e gera impacto e ineficiência no processo.

A manutenção adota a seguinte estratégia: para as paradas de emergência, existe uma equipe com as especialidades mecânica e elétrica para pronto atendimento. As manutenções preventivas ocorrem em paradas programadas, que na linha 1 acontecem mensalmente e nas linhas 2 e 3 bimestralmente. As paradas programadas normalmente duram 24 horas. A cada dois anos, uma parada programada de 10 dias é realizada, na qual são realizadas tarefas de manutenção de mais alcance e reforma de sub-sistemas recomendadas pelo fabricante e pela engenharia de manutenção da empresa. Em paralelo, atividades de manutenção preditiva utilizam técnicas de Análise de Vibração, Análise Termográfica, Análise de Óleos e Execução de Check-lists.

A manutenção também é responsável pelo atendimento e operação da área de Utilidades, que fornece outros insumos para as linhas de produção, tais como: água gelada, ar comprimido e óleo térmico.

\subsection{COLETA E MODELAGEM DOS DADOS}

Para o calculo da disponibilidade $A v(t)$ e localização na curva da banheira, foram utilizados os tempos para reparo $(T T R)$ e tempos até a falha $(T B F)$ para as três linhas. Estes dados são registrados diretamente pela produção e indicam as falhas que ocorrem ao longo do processo e interrompem o bobinamento de material na última etapa da transformação, no equipamento chamado Winder. Na coleta dos dados não foram consideradas falhas operacionais, manutenções programadas, testes de processo e problemas de utilidades. Os TTR e TBF coletados são exclusivamente de paradas emergenciais, 
ou seja, quebras de equipamentos ou subsistemas.

A data de inicio da coleta dos dados é a mesma para as três linhas, no início de abril de 2012. Foram coletados vinte eventos, registrados na Tabela 2.

\begin{tabular}{|c|c|c|c|c|c|c|c|c|c|}
\hline \multirow{2}{*}{$\begin{array}{c}\# \\
\text { falha }\end{array}$} & \multicolumn{3}{|c|}{ Linha 1} & \multicolumn{3}{|c|}{ Linha 2} & \multicolumn{3}{|c|}{ Linha 3} \\
\hline & data & $T T R$ & $T B F$ & data & $T T R$ & $T B F$ & data & $T T R$ & $T B F$ \\
\hline 1 & $04 / 04 / 2012$ & 4,2 & 83,5 & $04 / 04 / 2012$ & 4,3 & 113,4 & 03/04/2012 & 4,6 & 132,5 \\
\hline 2 & $04 / 04 / 2012$ & 2,0 & 4,8 & 04/04/2012 & 4,3 & 5,0 & $04 / 04 / 2012$ & 7,9 & 10,4 \\
\hline 3 & 05/04/2012 & 0,7 & 15,2 & 04/04/2012 & 4,0 & 1,2 & $10 / 04 / 2012$ & 2,0 & 141,6 \\
\hline 4 & 08/04/2012 & 1,2 & 67,3 & $05 / 04 / 2012$ & 3,5 & 8,2 & $11 / 04 / 2012$ & 4,7 & 29,4 \\
\hline 5 & $09 / 04 / 2012$ & 5,6 & 16,2 & $11 / 04 / 2012$ & 0,7 & 146,2 & $14 / 04 / 2012$ & 0,7 & 60,1 \\
\hline 6 & $12 / 04 / 2012$ & 1,2 & 69,0 & $14 / 04 / 2012$ & 7,5 & 64,7 & $16 / 04 / 2012$ & 4,2 & 48,5 \\
\hline 7 & $14 / 04 / 2012$ & 10,1 & 42,4 & $18 / 04 / 2012$ & 2,5 & 83,0 & $17 / 04 / 2012$ & 5,1 & 20,7 \\
\hline 8 & $15 / 04 / 2012$ & 2,5 & 14,5 & $19 / 04 / 2012$ & 5,1 & 12,2 & $20 / 04 / 2012$ & 1,4 & 70,0 \\
\hline 9 & $15 / 04 / 2012$ & 4,4 & 10,4 & $21 / 04 / 2012$ & 2,8 & 55,5 & $21 / 04 / 2012$ & 2,3 & 14,2 \\
\hline 10 & $17 / 04 / 2012$ & 3,4 & 26,2 & $28 / 04 / 2012$ & 2,8 & 152,2 & $23 / 04 / 2012$ & 4,8 & 50,1 \\
\hline 11 & 19/04/2012 & 2,8 & 50,1 & 29/04/2012 & 4,0 & 26,8 & $25 / 04 / 2012$ & 1,8 & 43,8 \\
\hline 12 & $24 / 04 / 2012$ & 4,0 & 122,8 & $30 / 04 / 2012$ & 3,9 & 15,0 & $27 / 04 / 2012$ & 3,9 & 55,1 \\
\hline 13 & $25 / 04 / 2012$ & 4,1 & 10,9 & 04/05/2012 & 4,2 & 95,4 & $02 / 05 / 2012$ & 5,7 & 106,4 \\
\hline 14 & $25 / 04 / 2012$ & 3,3 & 50,0 & 08/05/2012 & 0,4 & 102,7 & $04 / 05 / 2012$ & 1,0 & 37,7 \\
\hline 15 & $27 / 04 / 2012$ & 3,0 & 20,9 & $12 / 05 / 2012$ & 3,3 & 91,6 & 05/05/2012 & 4,1 & 23,6 \\
\hline 16 & $28 / 04 / 2012$ & 2,7 & 59,4 & $13 / 05 / 2012$ & 2,7 & 27,1 & $10 / 05 / 2012$ & 2,6 & 124,7 \\
\hline 17 & 01/05/2012 & 0,9 & 54,2 & $18 / 05 / 2012$ & 0,8 & 108,2 & $15 / 05 / 2012$ & 4,8 & 108,1 \\
\hline 18 & 03/05/2012 & 3,4 & 40,9 & 19/05/2012 & 6,8 & 17,9 & $17 / 05 / 2012$ & 2,9 & 56,5 \\
\hline 19 & $05 / 05 / 2012$ & 1,9 & 80,3 & $20 / 05 / 2012$ & 1,9 & 26,6 & $19 / 05 / 2012$ & 5,0 & 30,7 \\
\hline 20 & 08/05/2012 & 5,2 & 44,3 & $25 / 05 / 2012$ & 1,0 & 117,4 & $20 / 05 / 2012$ & 0,9 & 24,4 \\
\hline
\end{tabular}

Tabela 2 - Dados coletados em horas

Fonte: banco de dados da empresa

Para modelar os TTR e TBF, foi usado o software Proconf 2000. O software usa o método da máxima verossimilhança, descrito analiticamente em Fogliatto e Ribeiro (2009), traça os papéis de probabilidade e, pelos testes KS e Quiquadrado, aponta as distribuições que não podem ser rejeitadas. Cabe ao pesquisador indicar qual delas será adotada para descrever a $R(t)$, segundo uma teoria apropriada ao caso. Foi adotada a premissa de pesquisa mencionada na revisão. $\mathrm{O}$ Proconf 2000 também estima os parâmetros das distribuições, entre estes os valores esperados (MTBF e MTTR). Com os MTTR e MTBF são calculadas as disponibilidades $A v(t)$.

Ao fim, é apresentada uma tabela com o resumo dos resultados $\mathrm{e}$ considerações comuns aos três casos. 


\subsection{LINHA 1}

Para os TTR, o Proconf 2000 apresentou os resultados da Tabela 3. Pela premissa de pesquisa, escolheu-se a distribuição Lognormal. A Figura 3 apresenta o papel de probabilidade para a distribuição. A Figura 4 apresenta $M(t)$ para a linha 1 .
Para os TBF, o Proconf 2000 apresentou os resultados da Tabela 4. Pela premissa de pesquisa, escolheu-se a distribuição de Weibull. A Figura 5 apresenta o papel de probabilidade para a distribuição. A Figura 6 apresenta $R(t)$ para a linha 1.

Tabela 3 - Testes para os TTR da linha 1

\begin{tabular}{|c|c|c|c|c|}
\hline \multicolumn{5}{|c|}{ Linha 1 - TTR } \\
\hline & MTTR & Dados significativos & Faixa $M T T R$ & Significância \\
\hline Lognormal & 3,3878 & $\mathrm{t} 10=1,21 ; \mathrm{t} 50=2,76$ & 2,47 até 4,73 & 0,2181 \\
\hline Exponencial & rejeitada & & & \\
\hline Normal & rejeitada & & & \\
\hline Weibull & 3,3021 & distrib & não considerada & \\
\hline Gamma & 3,4059 & distrib & não considerada & \\
\hline
\end{tabular}

Fonte: Proconf 2000

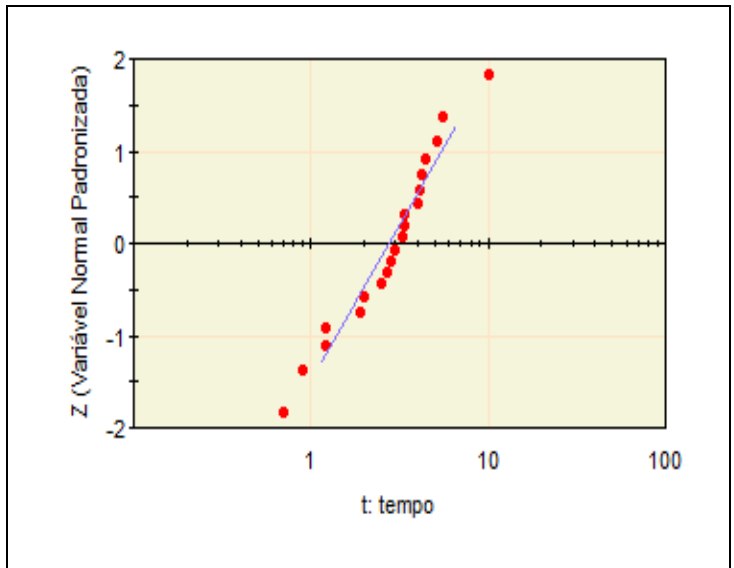

Fig. 3 - Papel de Probabilidade Lognormal para os TTR da linha 1

Fonte: Proconf 2000

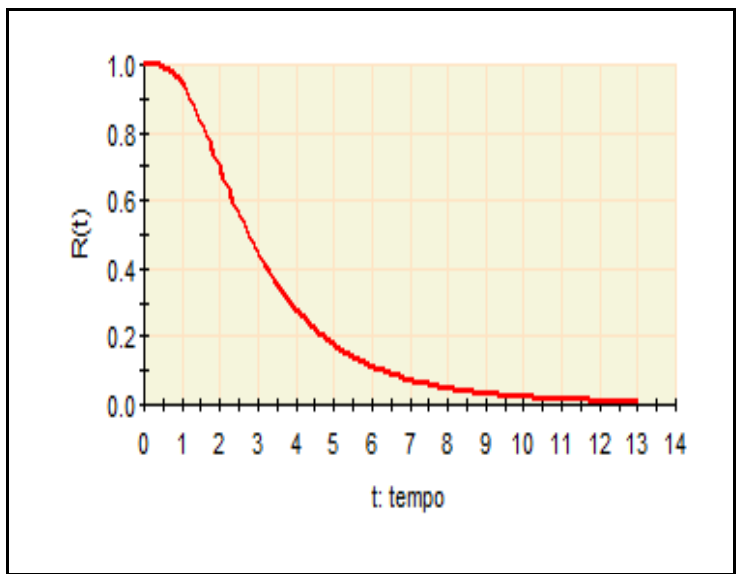

Fig. 4 - Função Mantenabilidade para a linha 1, em horas

Fonte: Proconf, 2000

Tabela 4 - Resumo dos testes de distribuição para os $T B F$ da linha 1

\begin{tabular}{|c|c|c|c|c|}
\hline \multicolumn{5}{|c|}{ Linha $1-T B F$} \\
\hline & $M T B F$ & Dados significativos & Faixa $M T B F$ & Significância \\
\hline Weibull & 43,5797 & fator de forma $=1,34$ & 32,30 até 68,76 & 0,9413 \\
\hline Exponencial & rejeitada & \multirow{2}{*}{\multicolumn{3}{|c|}{ distribuição não considerada }} \\
\hline Gamma & 45,5764 & & & \\
\hline Lognormal & rejeitada & \multirow{2}{*}{\multicolumn{3}{|c|}{ distribuição não considerada }} \\
\hline Normal & 44,045 & & & \\
\hline
\end{tabular}

Fonte: Proconf, 2000 


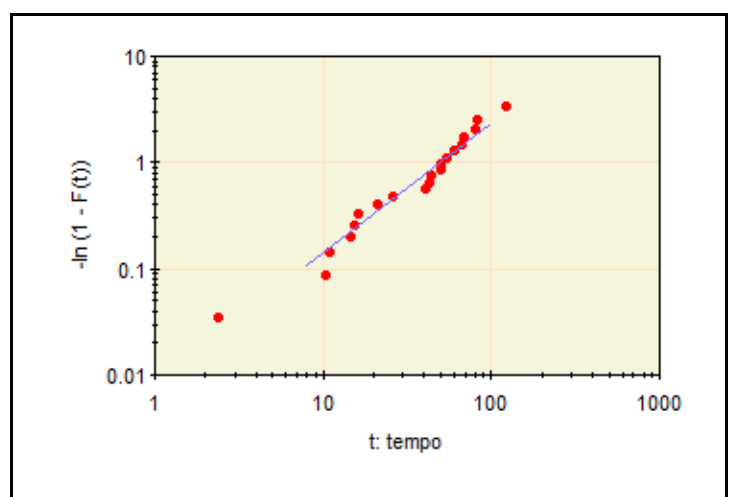

Fig. 5 - Papel de Probabilidade Weibull para os TBF da linha 1

Fonte: Proconf, 2000

Com os valores de MTBF e MTTR encontrados, calculou-se a disponibilidade $A v(t)$ da linha 1, utilizando a equação (18). Pode-se dizer que em $92,78 \%$ do tempo, da perspectiva da manutenção, o equipamento esteve disponível para produção.

$$
\begin{aligned}
& A v(t)=\frac{M T B F}{M T B F+M T T R}=\frac{43,5797}{43,5797+3,3878}= \\
& A v(t)=92,79 \%
\end{aligned}
$$

\subsection{LINHA 2}

Para os TTR, o Proconf 2000 apresentou os resultados da Tabela 5. Pela premissa de pesquisa, escolheu-se a distribuição normal. A Figura 7 apresenta o papel de probabilidade para a distribuição. A Figura 8 apresenta a $M(t)$ para a linha 2.

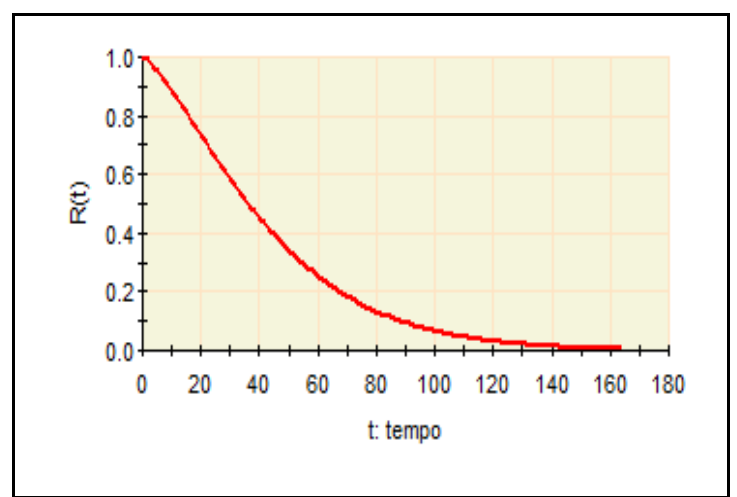

Fig. 6 - Função Confiabilidade para a linha 1 , em horas

Fonte: Proconf, 2000

Para os $T B F$, o Proconf 2000 apresentou os resultados da Tabela 6. Pela premissa de pesquisa, escolheu-se a distribuição exponencial. A Figura 9 apresenta o papel de probabilidade para a distribuição. A Figura 10 apresenta $R(t)$ para a linha 2 .

Tabela 5 - Resumo dos testes de distribuição para os TTR da linha 2

\begin{tabular}{ccccc}
\hline \multicolumn{5}{c}{ Linha $2-T T R$} \\
\hline Normal & MTTR & Dados significativos & Faixa $M T T R$ & Significância \\
\hline Exponencial & rejeitada & t10 $=1,24 ;$ t50 $=3,41$ & 2,44 até 4,2 & 0,1657 \\
Lognormal & rejeitada & & \\
Weibull & 3,2756 & distribuição não considerada & \\
Gamma & 3,4066 & distribuição não considerada & \\
\hline
\end{tabular}

Fonte: Proconf, 2000 


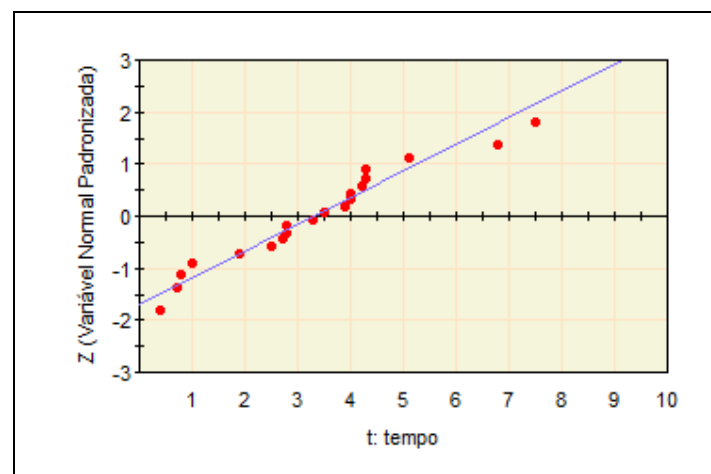

Fig. 7 - Papel de Probabilidade Normal para os TTR da linha 2

Fonte: Proconf, 2000

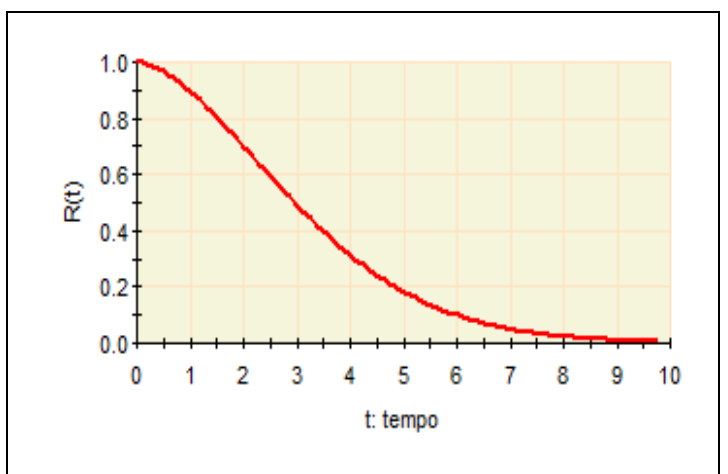

Fig. 8 - Função Mantenabilidade da linha 2, em horas

Fonte: Proconf, 2000

Tabela 6 - Resumo dos testes de distribuição para $T B F$ linha 2

\begin{tabular}{ccccc}
\hline & \multicolumn{4}{c}{ Linha $2-T B F$} \\
\hline Exponencial & MTBF & Dados significativos & Faixa $M T B F$ & Significância \\
Weibull & rejeitada & taxa de falha $=0,0157$ & 43,17 até 105,87 & 0,0844 \\
Gamma & rejeitada & & & \\
Lognormal & rejeitada & & & \\
Normal & rejeitada & & & \\
\hline
\end{tabular}

Fonte: Proconf, 2000

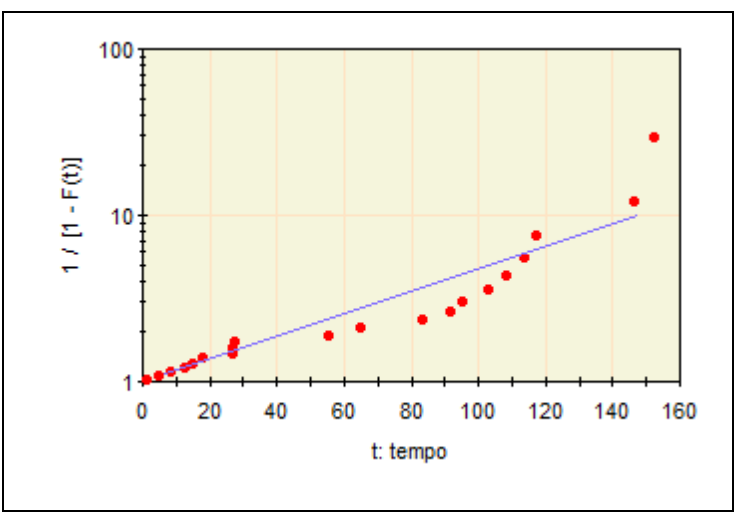

Fig. 9 - Papel de Probabilidade Exponencial para os $T B F$ da linha 2 Fonte: Proconf, 2000

Com os valores de MTBF e MTTR encontrados pode-se calcular a disponibilidade $A v(t)$ da linha 2, utilizando a equação (19). Pode-se dizer que em $95,02 \%$ do tempo, da perspectiva da

\subsection{LINHA 3}

Para os TTR, o Proconf 2000 apresentou os resultados da Tabela 7. Pela premissa de pesquisa, escolheu-se a

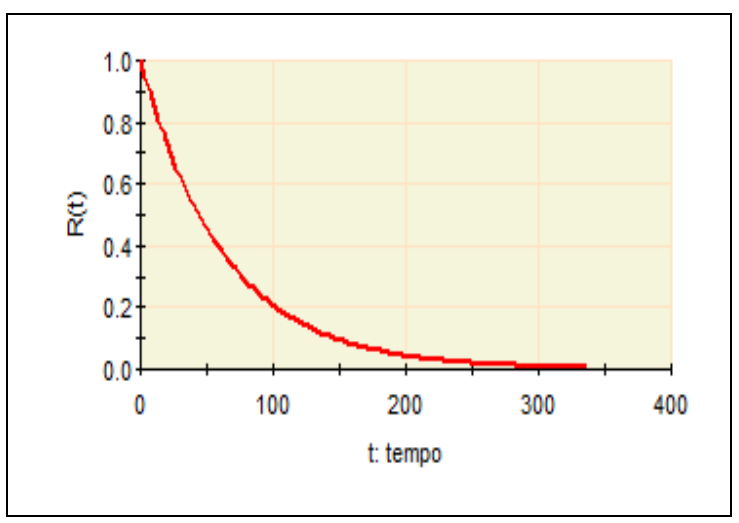

Fig. 10 - Função Confiabilidade da linha 2, em horas

Fonte: Proconf, 2000

manutenção, o equipamento esteve disponível para produção.

$$
\begin{aligned}
& A v(t)=\frac{M T B F}{M T B F+M T T R}=\frac{63,515}{63,515+3,325}= \\
& =95,03 \%
\end{aligned}
$$

distribuição normal. A Figura 11 apresenta o papel de probabilidade para a distribuição. A Figura 12 apresenta a $M(t)$ para a linha 3 . 
Tabela 7 - Resumo dos testes de distribuição para TTR linha 3

\begin{tabular}{ccccc}
\hline \multicolumn{5}{c}{ Linha $3-T T R$} \\
\hline Normal & $M T T R$ & Dados significativos & Faixa $M T T R$ & Significância \\
\hline Exponencial & 3,52 & $\mathrm{t} 10=1,36 ; \mathrm{t} 50=3,59$ & 2,62 até 4,41 & 0,287 \\
Lognormal & rejeitada & & & \\
Weibull & rejeitada & distribuição não considerada & \\
Gamma & 3,4772 & distribuição não considerada & \\
\hline
\end{tabular}

Fonte: Proconf, 2000

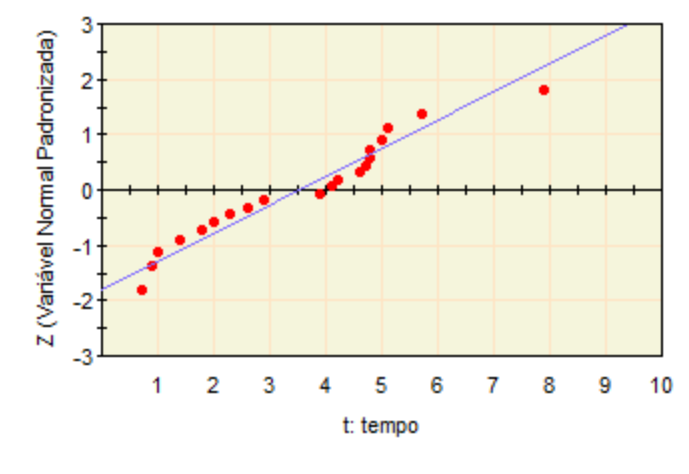

Fig. 11 - Papel de Probabilidade Normal para os TTR da linha 3

Fonte: Proconf, 2000

Para os TBF, o Proconf 2000 apresentou os resultados da Tabela 8. Pela premissa de pesquisa, escolheu-se a distribuição de Weibull. A Figura 13

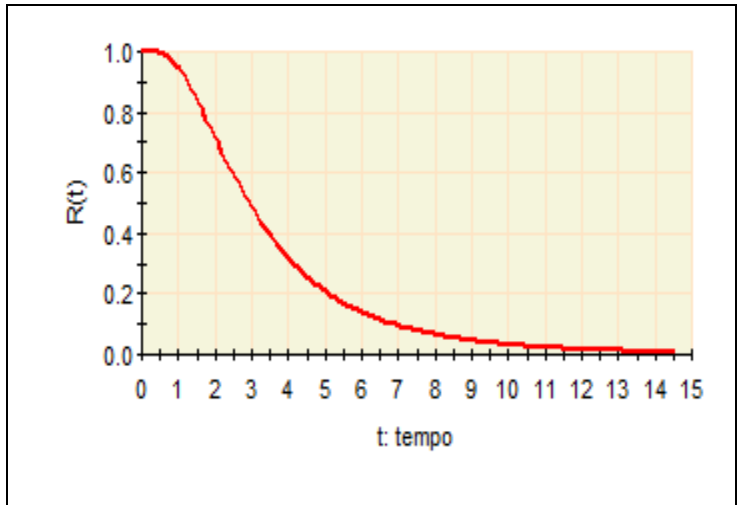

Fig. 12 - Função Mantenabilidade para a linha 3, em horas

Fonte: Proconf, 2000

apresenta o papel de probabilidade para a distribuição. A Figura 14 apresenta $R(t)$ para a linha 3 .

Tabela 8 - Resumo dos testes de distribuição para os $T B F$ da linha 3

\begin{tabular}{ccccc}
\hline \multicolumn{5}{c}{ Linha 3 - TBF } \\
\hline Weibull & MTBF & Dados significativos & Faixa $M T B F$ & Significância \\
\hline Exponencial & 59,1027 & fator de forma $=1,2698$ & 38,43 até 85,56 & 0,2418 \\
Gamma & rejeitada & & & \\
Lognormal & 61,3432 & distribuição não considerada & \\
Normal & 60,6644 & distribuição não considerada & \\
\hline
\end{tabular}

Fonte: Proconf, 2000 


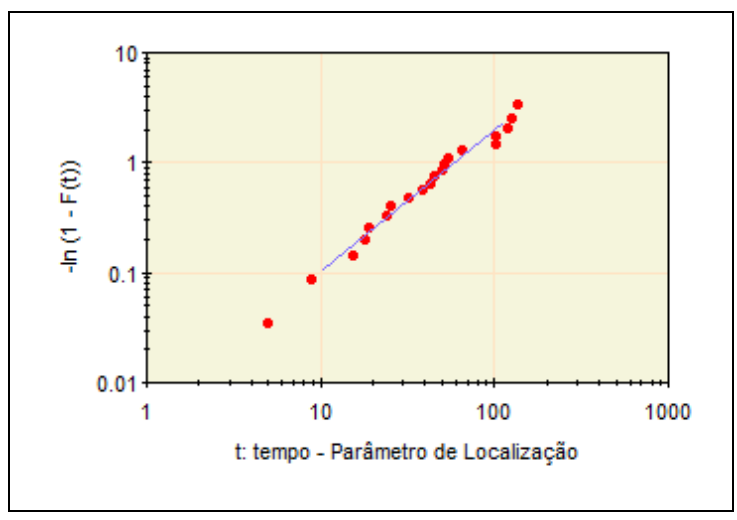

Fig. 13 - Papel de Probabilidade Weibull para os TTR da linha 3

Fonte: Proconf, 2000

Com os valores de MTBF e MTTR encontrados pode-se calcular a disponibilidade $A v(t)$ da linha 3, utilizando a equação (20). Pode-se dizer que em $94,37 \%$ do tempo, da perspectiva da manutenção, o equipamento esteve disponível para produção.

$$
\begin{aligned}
& A v(t)=\frac{M T B F}{M T B F+M T T R}=\frac{59,1027}{59,1027+3,52}= \\
& A v(t)=94,38 \%
\end{aligned}
$$

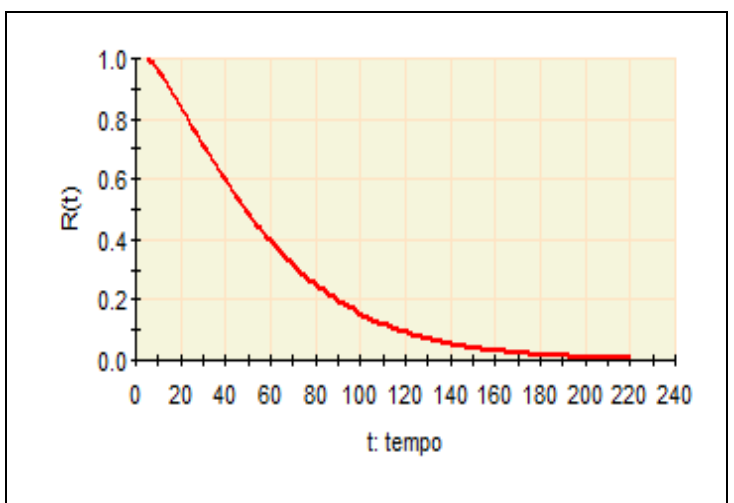

Fig. 14 - Função Confiabilidade para a linha 3, em horas Fonte: Proconf, 2000

\section{DISCUSSÃO}

Ao aplicar técnicas quantitativas da MCC para o cálculo da disponibilidade $A v(t)$ e identificação do posicionamento no ciclo de vida de três linhas destinadas a produção de BOPP, pode-se chegar a algumas conclusões. Os principais resultados encontrados estão agrupados na Tabela 9. A Figura 15 indica a posição na curva da banheira para as três linhas estudadas.

Tabela 9 - Síntese das três linhas

\begin{tabular}{rccc}
\hline & LINHA 1 & LINHA 2 & LINHA 3 \\
\hline Distribuição para TTR & Lognormal & Normal & Normal \\
MTTR (horas) & 3,3878 & 3,325 & 3,52 \\
Distribuição para TBF & Weibull & Exponencial & Weibull \\
MTBF (horas) & 43,5797 & 63,515 & 59,1027 \\
& Mortalidade & Maturidade & Mortalidade \\
Curva da Banheira & Senil & Senil \\
& $92,79 \%$ & $95,03 \%$ & $94,38 \%$ \\
\hline
\end{tabular}

Fonte: Proconf, 2000 


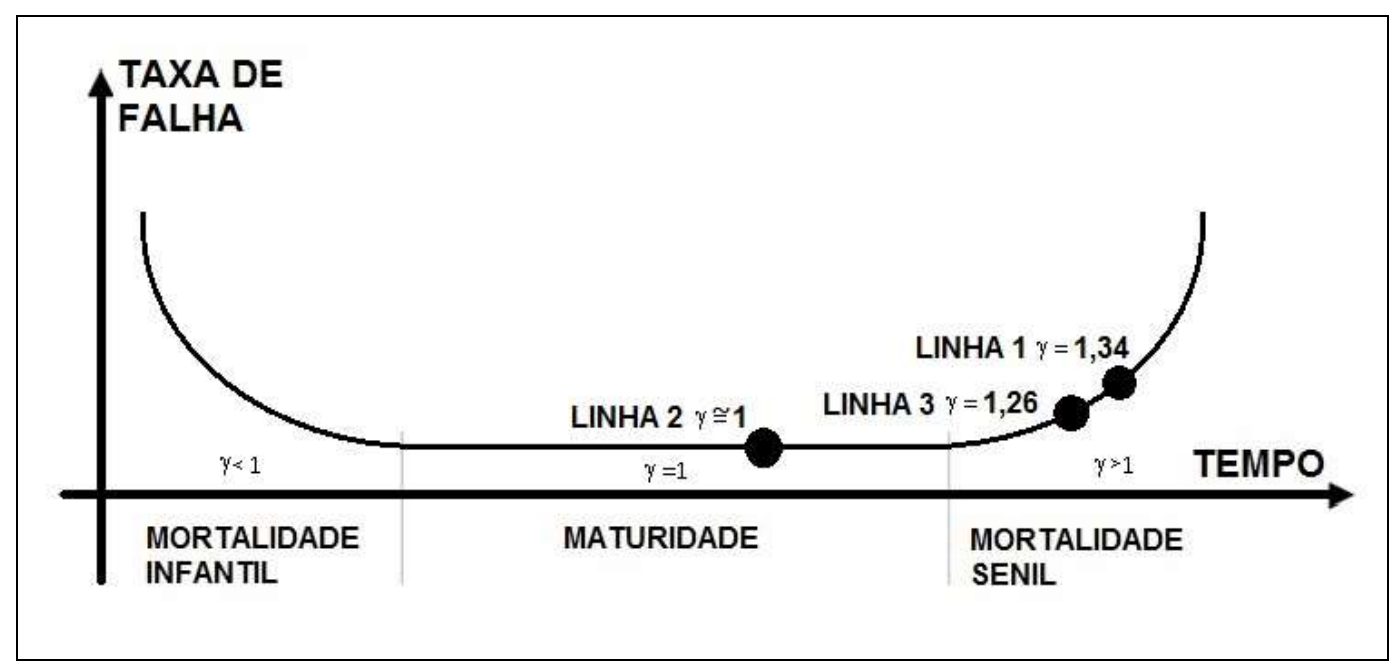

Fig. 15 - Posição na curva da banheira das três linhas

Conclui-se que a linha 2, apesar de não ser a mais nova, está na melhor condição do ponto de vista da confiabilidade e mantenabilidade, pois tem a maior disponibilidade e encontra-se na fase da maturidade. Nesta fase, a melhor estratégia de manutenção é a preditiva, se possível acompanhada de um programa organizacional de MPT (Manutenção Produtiva Total) (Sellitto, 2005).

A linha 1 apresentou os piores resultados em confiabilidade (o menor $M T B F)$, a mais baixa disponibilidade e encontra-se na pior posição da curva da banheira. Uma hipótese para esta posição é o maior tempo de uso do equipamento e também a tecnologia mais antiga, notadamente baseada em sistemas mecânicos sujeitos ao desgaste. As linhas 2 e 3, e principalmente a 3, já utilizam soluções baseadas e tecnologias eletrônicas que simplificam os sistemas mecânicos e reduzem os desgastes. Para as linhas 2 e 3 , a melhor estratégia de manutenção é a preventiva, acompanhada de reformas e retrofits para atualização tecnológica (Sellitto, 2005). Quanto à mantenabilidade, a linha 1 teve um MTTR melhor que o da linha 3.

A linha 3 apresenta um resultado abaixo do que se esperava, pois esta é a linha mais nova e com as soluções tecnológicas mais atualizadas. Em mantenabilidade, esta linha apresentou o pior MTTR e está posicionada na fase de mortalidade senil da curva da banheira, o que pode ser preocupante para uma linha com apenas cinco anos de uso. A mantenabilidade baixa pode ser explicada pela maior complexidade tecnológica, que, dado que uma falha ocorra, exige mais esforço dos técnicos para o reparo e peças com as quais a empresa não está familiarizada, tanto no manuseio como quanto à necessidade de estocagem. Em confiabilidade e disponibilidade esta linha tem resultados inferiores a linha 2, que já tem oito anos de uso. Para esta linha devese adotar a estratégia preventiva e também analisar de modo mais profundo porque se tem uma taxa de falhas crescente no equipamento que deveria estar com o melhor desempenho. Também é necessário mais treinamento dos técnicos de manutenção e eventualmente mais peçasreserva, pois alguns dos reparos mais longos estiveram ligados à falta de materiais adequados para reposição imediata. Tal fato deveu-se a que a empresa não percebeu de imediato a necessidade de se manterem estoques de peças de difícil aquisição.

Uma observação que é comum às três linhas é que todas já saíram do período de mortalidade infantil, evidenciando a qualidade de projeto dos equipamentos adquiridos e que possíveis problemas iniciais e de montagem já foram solucionados pela equipe de manutenção da empresa.

\section{CONSIDERAÇÕES FINAIS}

O objetivo deste artigo foi calcular a disponibilidade de três linhas de produção de BOPP (Película de 
Polipropileno Biorientada) em uma empresa da indústria química. Os objetivos secundários foram modelar as funções confiabilidade e manutenibilidade, posicionar as linhas no ciclo de vida, e então calcular as disponibilidades das três linhas estudadas. As principais técnicas de pesquisa foram a modelagem dos tempos entre falhas ( $T B F$ - time between failures) e tempos até o reparo (TTR - time to repair). Não foi objetivo do artigo analisar qualitativamente as causas para os tipos de falhas modelados nem estratégias para a gestão dos resultados encontrados. A escolha das três linhas deveu-se ao fato que as mesmas esgotam a capacidade produtiva da empresa. Deste modo, toda a instalação é estudada.

Concluiu-se que os dados foram originados do histórico de falhas mantido pela Manutenção da empresa estudada. Observa-se a importância da fidedignidade do banco de dados, o que se coaduna com o argumento usado por Fogliatto e Ribeiro (2009) acerca da importância dos registros de falhas em estudos de confiabilidade.

Como continuidade de pesquisa, sugere-se análise qualitativa acerca do tipo de falha que tem ocorrido, do tipo de qualificação dos manutentores e da qualidade da execução dos procedimentos de manutenção preventiva e preditiva. Também pode-se analisar qualitativamente a tecnologia empregada nas linhas e se eventualmente a diferença de tecnologia pode explicar a diferença de resultados, ou se os mesmos são decorrência da evolução ao longo da curva da banheira. Mesmo que os MTTR das três linhas sejam próximos, o $M T B F$ varia e, em decorrência, também varia a disponibilidade $A V$.

\section{REFERÊNCIAS}

Associação Brasileira De Normas Técnicas - ABNT (1994) NBR 5462. Confiabilidade e mantenabilidade. Rio de Janeiro.

BRUCKNER. 2013. Manual de Operações. disponível em http://www.brueckner.com/en/bruecknermaschinenbau/solutions/packaging-filmlines/bopp/, acesso em 06-04-2013.
DODSON, B.; NOLAN, D. 2002. Reliability Engineering Handbook. New York: John Wiley \& sons.

FOGLIATTO, F.; RIBEIRO, J. 2009. Confiabilidade e Manutenção Industrial. Rio de Janeiro: Elsevier.

HAHN, G.; SHAPIRO, S. 1967. Statistical models in engineering. New York: John Wiley \& Sons.

LAFRAIA, J. 2001. Manual de Confiabilidade, Mantenabilidade e Disponibilidade. Rio de Janeiro: Qualitymark.

LAWLESS, J. 2003. Statistical models and methods for lifetime data. New York: John Wiley \& Sons.

LEWIS, E. 1996. Introduction to Reliability Engineering. New York: John Wiley \& Sons.

MENDES, A. 2011. Manutenção Centrada em Confiabilidade: uma abordagem quantitativa. Dissertação (Mestrado em Engenharia de Produção), Universidade Federal do Rio Grande do Sul - UFRGS, Porto Alegre.

MENGUE, D.; SELLITTO, M. 2013. Estratégia de manutenção baseada em funções de confiabilidade para uma bomba centrífuga petrolífera. Produção Online, v.13, n.2, p. 759-783.

PINTO, A.; XAVIER, J. 1999. Manutenção: função estratégica. Rio de Janeiro: Qualitymark.

PROCONF. 2000. Confiabilidade de componentes: Software. Porto Alegre: Copyright $\odot$, Maxxi Gestão Empresarial.

SELLITTO, M. 2005. Formulação estratégica da manutenção industrial com base na confiabilidade dos equipamentos. Produção, v.15, n.1, p.44-59.

SELLITTO, M.; BORCHARDT, M.; ARAÚJO, D. Manutenção centrada em confiabilidade: aplicando uma abordagem quantitativa. Anais do XXII ENEGEP, 
Encontro Nacional de Engenharia de Produção. 2002. Curitiba.

SELLITTO, M. 2002. Inteligência artificial: uma aplicação em uma indústria de processo contínuo. Gestão \& Produção, v.9, n.3, p.363-376.

SIQUEIRA, I. 2005. Manutenção centrada em confiabilidade. Rio de Janeiro: Qualitymark.
SWANSON, L. 2001.Linking maintenance strategies to performance. International Journal of Production Economics, v.70, n. 3, p.237-244.

WUTTKE, R.; SELLITTO, M. 2008.Cálculo da disponibilidade e da posição na curva da banheira de uma válvula de processo petroquímico. Produção Online, v.8, n.4, p.1-23. 